كلمة التحرير

\title{
رؤية العالم
}

\section{فنحي حسن ملكاوي}

يأتي هذا العدد الأربعون من "إسلامية المعرفة" -المجلة- لتكمل به سنتها العاشرة من عمرها، الذي نرجو أن يمتد طويلاً، حتى تسهم مع غيرها من الوسائل في تحقيق "إسلامية المعرفة" -الهدف- الذي يمثل بُعداً مهماً من أبعاد الإصلاح الإسلامي في بجال الفكر والثقافة. والعلاقة وثيقة بين بجالات الإصلاح السياسي والاقتصادي والاجتماعي والثقافي؛ فالجدل حول أولويات الإصلاح في هذه البمالات لا طائل من ورائه؛ لأن المسؤولية في فاية المطاف -أمام الناس والتاريخ" هي مسؤولية كل فئات الأمة في تحقيق غايات الإصلاح

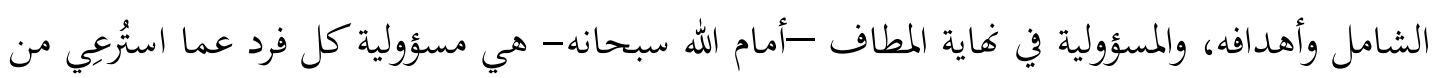
شؤون مجتمعه وأمتّه، ليتحقق الإحسان في رعاية هذه الشؤون، ومن ثم ليتحقق صلاح المجتمع في الدنيا وخلاص الفرد في الآخرة. ومن هنا جاء فقه الثغرة، وفقه السفينة.

إسلامية المعرفة- المجلة وسيلة من وسائل تحقيق أهداف الإصلاح الفكري والمنهجي؛ وهي منبر متخصص

للحوار حول الأفكار الإصلاحية والتجديدية، ومنتدى لعرض نتائج البحوث والدراسات حول قضايا الإصلاح. وقد يكون من المفيد أن نذكّر بين الحين والآخر بأن قضايا البحث التي تتخصص المجلة في نشرها تتوزع في أربعة محاور:

•الرؤية الكلية وتشتمل علي قضيتين هما النظام المعري والتفكير المنهجي،

•منهجية التعامل مع الأصول: وتشتمل على قضيتين هما: منهجية التعامل مع القرآن الكريم ومنهجية التعامل مع السنة النبوية الشريفة،

•منهجية التعامل مع التراث: وتشتمل على قضيتين هما: منهجية التعامل مع التراث الإسلامي ومنهجية

$$
\text { التعامل مع التراث الإنساني، }
$$

•منهجية التعامل مع الواقع: وتشتمل على قضيتين هما: منهجية التعامل مع الواقع كما هو، ومنهجية التعامل مع الواقع كما يجب أن يكون، ونعني بذلك الدراسات المستقبلية والاستشرافية. 
والتذكير بهذه المحاور والقضايا الكبرى هو للتأكيد، مرة بعد أخرى، على الطبيعة المتخصصة للمجلة التي تنطلق من الرؤية الكلية هدفاً وموضوعاً، وتعتمد المعالجة المنهجية طريقة وأسلوباً. وسوف نستطرد في هذا المقام شيئاً قليلاً حول ما نقصده ب "الرؤية الكلية" تاركين النفصيل إلى مقام آخر.

يتشابه الناس في الخصائص المادية والنفسية والعقلية، ولكنهم أيضاً يختلفون في هذه الخصائص وغيرها، فما المهم فيما يختلف الناس فيه أو يتشاهون؟ عند النظر إلى التفاصيل نجد أن الناس يختلفون في أشياء كثيرة جداً، ويمكن لأي شيء أن يكون في لحظة معينة العنصر المهم في تحديد الاختلاف، وفي لحظة أخرى ينحسر هذا العنصر ليفسح بجالاً لعنصر آخر يعبر عن الاختلاف بصورة أكثر أهمية. وهكذا تبدو التفاصيل غير قادرة على بيان المهم والأكثر أهمية في تحديد جوهر الاختلاف بين الناس. من هنا تأتي أهمية البحث عن

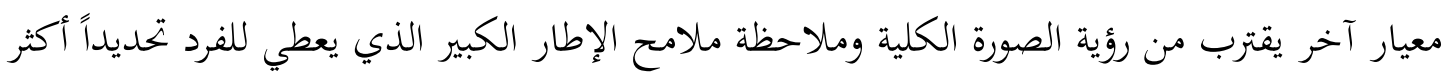
تعبيراً، ووصفاً أدق دلالة من تلك العناصر التفصيلية. من المعروف أن ما يراه الإنسان بعينيه ويسمعه بأذنيه يعتمد إلى حد كبير على الموقع الذي يرى منه الأشياء ويسمعها، كما يعتمد على هويته التي تحدد أي نوع من الناس هو. لكن من المعروف أيضاً أن معتقدات الإنسان المعلنة وسلوكاته الملحوظة في سائر الظواهر الثقافية والاجتماعية هي في حقيقة الأمر متجذرة بطريقة واعية أو غير واعية في بعض المفاهيم والمبادئ الأكثر عمقاً في حياة الناس أو تعبيراً عنها. ومع أن هذه المفاهيم والمبادئ العامة يتم التعبير عنها بوساطة مصطلحات ومفردات لغوية، فإن كلا منها لا بقوم وحده، ولا تتحدد دلالثه الكاملة منفصلاً عن غيره، فدلالات هذه المصطلحات والمفردات اللغوية متداخلة جداً، وتشتق معانيها من نظام واسع من العلاقات المتشابكة، الشديدة التعقيد. إنه النظام الفكري -الجشتالتيالكلي الذي تتحدد ضمنه المعاني الكامنة والدلالات الحقيقية لهذه المصطلحات . إن العلاقة بين رؤية التفاصيل والرؤية الكلية ليست علاقة سطحية خطية، وإنما هي علاقة تفاعلية جدلية معقدة، فالرؤية الكلية تضع التفاصيل في موقعها المناسب، وإدراك التفاصيل في حدودها الخاصة بها لا يقتصر على التقاطها وتمييزها في موقعها من الزمان والمكان، وإنما يتجاوز ذلك إلى ربطها برموزها ودلالاتها وما يرتبط بها من أفعال وردود أفعال، وهذا يعني الارتقاء بها إلى مستوى من التجريد الدلالي الذي يتطلب 
بالضرورة إعمال العقل والتأمل العميق، مما ينتج عنه تعديل في الرؤية الكلية وتوسيع في بجالاتما . ويتداخل مفهوم الرؤية الكلية مع بعض الأدوات المنهجية المعرفية التي يستخدمها المفكر في تحليل الظواهر والوقائع والأفكار، همدف تمكينه من رؤية كلية للموضوعات المتفرقة، فتربط الكلي والجزئي، والعام والخاص، حتى تتحقق الإحاطة بالظاهرة موضوع الدراسة والإدراك الشمولي لها. ومن هذه الأدوات المنهجية ما يسميه عبد الوهاب المسيري "النموذج التفسيري" الذي يتحدد في "جموعة من الصفات التي تحولت إلى صورة متماسكة، ترسخت في أذهاننا ووعينا بحيث لا نرى الواقع إلا من خلالها، فهي رؤية متكاملة للواقع." هذا النموذج التفسيري هو خريطة معرفية يْنيها العقل الإنساني، ويتوصل إليها عن طريق بتريدكمٍ هائل من العلاقات والتفاصيل والحقائق والربط بينها لبناء نمط عام يأخذ شكل خريطة إدراكية كلية. هذا "النموذج الإدراكي" هو أداة الإنسان في إدراك الواقع، ولكنه يتم "في أغلب الأحيان بصورة غير واعية يستنبطها المرء تدريجياً وتصبح جزءاً من وجدانه وسليقته وإدراكه المباشر، من خلال ثقافته وتفاصيل حياته، وما يتشكل منه لهن عالمه من أشياء ورموز وعلامات وصور وأحلام ومنتجات حضارية متعددة." ويتزاوج مع النماذج الإدراكية "نماذج تحليلية" إبداعية واعية يصوغها الباحث من خلال قراءاته للنصوص المختلفة، وملاحظته للظواهر، ثم يقوم بتفكيك الواقع وإعادة تركيبه من خلالها، بحيث يصبح الواقع أو النص مفهوماً ومستوعباً بشكل أعمق. وتعمل النماذج التحليلية على توسيع نطاق النموذج التفسيري من خلال الظواهر والمعطيات التي يحاول النموذج أن يفسرها. فهذه المعطيات تتحدى النموذج وتكشف قصوره، وربما يلزم تعديل النموذج حتى تزداد قدرته التفسيرية، وهكذا فإن العلاقة بين النموذج التفسيري والواقع علاقة حلزونية معقدة . إنّ الصورة الكلية التي يكوها الإنسان لنفسه عن نفسه وعن العالم من حوله، في حدود الموقع الذي يحاول منه الرؤية، وزاوية النظر التي يتخذها، والبيئة الطبيعية والنفسية والاجتماعية، والنظام الفكري بمكوناته اللغوية وأطره المرجعية... هذه الصورة الكلية هي التي تعرّف الإنسان، عندما تنظر إليه من الخارج، وتعرّفه برؤيته هو لنفسه ولأشياء من حوله، وهي ما يعرف بالرؤية الكلية، أو الرؤية الكونية، أو الفكرة الكلية، أو التصور الكلي، أو الفلسفة العامة، أو التفسير الشامل، أو النموذج التفسيري أو الإيديولوجيا... أو ما أصبح يعرف على نطاق واسع: رؤية العالم. ويتداخل مفهوم رؤية العالم في مختلف حقول المعرفة: في الدين، والفلسفة، والعلوم الاجتماعية والطبيعية، 
والفنون، والعلوم التطبيقية مثل الطب والهندسة... الخ. فالمصطلحات الدينية مثل الإيمان والعقيدة والتصور الكلي تعبر عن مجموعة الأفكار والمفاهيم والمعتقدات التي تجيب عن الأسئلة الوجودية الكبرى التي يحاول مصطلح "رؤية العالم" التعرض لها. وهي نفسها الأسئلة التي انشغلت بها الفلسفة منذ بداية عهد الإنسان بميادينها. وهي المحتوى الأساسي لفلسفة أي علم من العلوم الحديثة الذي يؤثر في تشكيل نظريات هذه العلوم ومناهج البحث فيها. وجميع الأفلام السينمائية، من أكثرها إثارة للضحك (كوميدية) إلى أكثرها إثارة للحزن (تراجيدية)، رغم أها تأخذ بخيال المشاهد، إلا أغا تقدم قيماً ورؤى محددة للعالم؛ إذ لا يوجد فيلم واجد يعرض قصة محايدة، لا تكون مطبوعة بالمعتقدات والقيم الثقافية للمؤلف والممثل والمخرج · أي أن الموضوع يرتبط بالرغبة العميقة وبالحاجة الأصيلة من فطره الإنسان في البحث عن إجابات الأسئلة الكلية والغائية التي يطرحها وجود الإنسان وحياته وعلاقته بالكون الذي يعيش فيه؛ من أين جاء وإلى أين يصير؟ لكن أمر هذه الأسئلة لا يبقى مجرد إحساس فطري بل يتحول إلى جهود عقلانية منظمة تبني عليها نظريات ويلتزم وفقها بإيديولوجيات، ومن تَّمّ ينشئ الدول وينظم الأحزاب ويشرع في الحروب، ثم ينشئ المنظمات الدولية للسعي لحل النزاعات ونشر السلام وإقامة العدل.... ورغم المقولة المعروفة في استخدام المصطلحات: "لا مشاحة في الاصطلاح،" فإن بعض الناس لا يفضلون استخدام مصطلح لم يرد في نصوص القرآن الكريم أو ا لحديث النبوي الشريف. ومع أن تطور العلوم قد أضاف إلى مفردات اللغة كثيراً من المصطلحات التي استقرت لدى أهل العلم، في الدلالة على فئات العلوم: النافعة والضارة، المحمودة والمذمومة، النقليّة والعقلية، الشرعية (علوم الشريعة) وغير الشرعية (كل ما عدا علوم الشريعة) أو على علم معين: علم أصول الفقه، وعلم مصطلح الحديث، مع ذلك فثمة من يتحفظ على استخدام مصطلحات جديدة، فيرى أن مصطلح علم الإيمان يجب أن يستخدم بدلاً من علم العقيدة....الخ. ومن بين أسباب هذا التحفظ أن بعض المصطلحات ربما يحمل جذوراً وملابسات تشوش دلالة المصطلح، وتبعدها عن المفهوم الذي يريده الكاتب. فالمرحوم سيد قطب كان مشغولاً منذ مطلع الخمسينات بالربط بين الفهم الذي تتطلبه عقيدة الإسلام وما يبنى عليها من سلوك، والفكرة الكلية عن الكون والحياة والإنسان، وكان يعد بإفراد هذا الموضوع ببحث متخصص، إلى أن أخرج كتاب "خصائص التصور الإسلامي ومقوماته." وقد ناقش أهمية استخدام مصطلح محدد لهذا الغرض، يعبر عن "الفكرة الكلية عن 
الكون والحياة والإنسان." ولم يكتف باستخدام مصطلح العقيدة أو الفكرة الكلية، ولم يقبل بمصطلح الفلسفة الإسلامية الذي نوقشت عناصر الموضوع تحته، ومع ذلك كان لا بد من اختيار مصطلح محدد، فاختار مصطلح: "التصور." ولا شك في أن من المفيد والمهم كذلك معرفة جذور المصطلحات وتطورها الدلالي، وعلاقة ذلك بالوضوح الفكري (أو الفوضى الفكرية) وبخاصة في فترات التفاعل الثقافي بين الأمم والشعوب، مثلما يحصل في عصرنا هذا من عولمة للأفكار والممارسات، واقتراض متبادل للمفاهيم والمصطلحات. وهي فترات تخشى فيها الأمم الضعيفة من المصير الذي يتهلد هويتها: تاريناً ولغة وثقافة. ومن المتوقع أن الكاتب إذا أراد أن يتجنب استخدام مصطلح معين، خشية من ارتباط دلالته بخلفيات معينة، فإن اختياره لمصطلح آخر بديل لن ينقذ المصطلح الجديد من الخلفيات الثقافية الخاصة للكاتب نفسه. ويحاول بعض الكتاب أن يعوّم المصطلح فيستخدم ألفاظاً متقاربة وبصورة مترادفة، مؤكداً أن ما يقصده هو لا يختلف في الإطار العام عما يقصده غيره من أي من تلك الألفاظ الأخرى. وأحد أسباب اللجوء إلى هذه "الحيلة" أن الكاتب في السياق الذي يكتب فيه لا يكون بصدد التأصيل والتمييز والتفريق بين دلالات الألفاظ -رغم علمه بوجودها- وأنه لا يريد أن يفتقد متابعة القارئ في هذا السياق للفكرة التي يريد الوصول إليها. و أحياناً يكون الكاتب قد وضح الفروق في مقام آخر يشير إليه، أو يقرر أن تلك الفروق تحتاج إلى وقفة متأنية في بحث آخر يعِد القارئ به.

وعلى أية حال فإن الثقافات الإنسانية عرفت هجرة المصطلحات من ثقافة إلى أخرى، ومن ميدان معرفي إلى آخر. وحين يهاجر المصطلح فإن جهوداً تبذل في توطين المصطلح لكي يحمل في موطنه الجديد دلالات محددة لا تتقيد بالضرورة بجذوره الأولى. إن مفهوم رؤية العالم مفهوم مهاجر انتقل موطنه الأصلي في الفلسفة وتوطّن في عدد واسع من المجالات المعرفية وبخاصة في العلوم الطبيعية والعلوم الاجتماعية، وسوف لن نبالغ في تقدير أهمية مفهوم "رؤية العالم" إذا أدركنا درجة تأثير الطرق الأساسية الذي ندرك فيها العالم الذي نعيش فيه، وموقعنا فيه، في فهمنا للعلوم الطبيعية والاجتماعية. وقد أصبح مصطلح رؤية العالم شائعاً في العقود الثلاثة الأخيرة في جميع الكتابات التي تتعلق بالأسئلة الكبرى 
في الدين والفلسفة والعلوم، حتى أصبحت من المصطلحات التي لا يُستغنى عنها. ومع ذلك فقليل جداً من الكتابات تعرضت للتاريخ الفلسفي لهذا المصطلح. وتشير الدراسات إلى أن المفكرين الألمان اهتموا أكثر من غيرهم منذ وقت مبكر بدراسة تاريخ الألفاظ والمصطلحات وتاريخ المفاهيم والأفكار، وبخاصة منذ النصف الثاني من القرن التاسع عشر. وقد رصد ديفيد نوجل سبعة من المراجع المهمة التي صدرت منذ مطلع القرن العشرين بالألمانية حول تشريح مصطلح رؤية العالم وتطور استعماله. كما يلاحظ كيف أن هذا الموضوع لم ينل إلا القليل من الاهتمام في الكتابات باللغة الإنجليزية . إن وجود تعدد في الرؤى الكلية حول العالم هو أحد خصائص الثقافة المعاصرة. وإذا كانت العولمة في هذا العصر هي اتجاه آخذ في الانتشار لتعميم ثقافة واحدة، فإن الاختلاف والتعدد في رؤية العالم هو خاصية ميزة لمذا العصر أيضاً، وربما تخدم العولمة في جعل هذه الرؤى المتعددة معروفة ومتاحة لأعداد متزايدة من الناس. وهكذا يستخدم مفهوم رؤية العالم في هذه الأيام لتوضيح ظاهرة التنافس الثقافي بين رؤى فلسفية ودينية متعددة. وهي من ثم رؤية "ما بعد حداثية" تتصف بالتعلد المعريز والتناشز الأخلاقي . وفي أثناء فترة الحرب الباردة كان التنافس والصراع على أساس سياسي ونفوذ إيديولوجي ومصالح اقتصادية

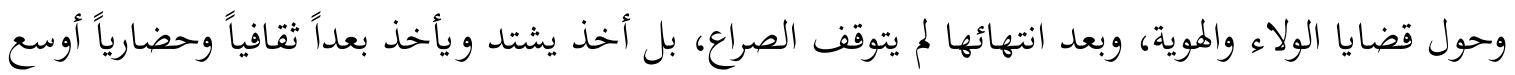
مدى وأعمق في جذوره، وأوسع في مجالاته، فالشعوب أصبحت تحاول الإجابة عن الأسئلة التي تواجهها، وبالذات الأسئلة الأكثر عمقاً، وأصبحت بجيب عنها بالطريقة التقليدية التي كانت هذه الشعوب تستعملها سابقاً؛ أي بالإشارة إلى الأشياء التي هي أكثر معنى من غيرها. والأشياء الأكثر معنى لأكثر الناس هي أجدادهم ولغتهم وتاريخهم وقيمهم وعاداتم ومؤسساقم التقليدية، وبخاصة الدين. وفي القلب من هذه الحرب الثقافية على المستوى المحلي والإقليمي والعالمي "صدام الرؤى الكونية." ويتجاوز هذا الصدام أحياناً الكلام ليصبح أكثر دموية، كما يتزايد الميل لتكون طرق التنافس في فهم الوجود الإنساني أكثر عنفاً، الأمر الذي يوضح أهمية البحث في مفهوم "رؤية العالم" أو "الرؤية الكونية." إن الصدام بين الطرق المختلفة في الوعي Collision of consciousness كان عاملاً محدداً لملحمة التاريخ البشري منذ عهود النشأة الأولى. فهو صدام حول المبادئ الأولى، فالأفكار لها نتائجها، ومع ذلك فإن ثمة مستوى عميقاً من إدراك الحقيقة يجب النظر إليه عند محاولة فهم الاختلاف الإيديولوجي الذي يقع 
في القلب من قصة الإنسان على هذه الأرض. ففي الوعي الديني (على الأقل في الأديان التوحيدية والوثنية) يحتل الصدام بين رؤى العالم موقعاً مهماً في المعركة الروحية المفية بين الحق والباطل، بين طاعة الله وطاعة الشيطان، وهي معركة تدور رحاها في عقول الناس وقلوبهم، ومن ثم في حياقم ومصيرهم، منذ بدء الوجود البشري. وليس ثمة ما هو أكثر أهمية من الطريقة التي يفهم فيها الإنسان خالقه ونفسه والكون الذي يعيش فيه وموقعه فيه.

كتب محرر إحدى دور النشر الأمريكية الدكتور جيمس سير James W. Sire كتاباً بعنوان "الكون المجاور لنا: دليل أساسي لرؤى العالم"، ونشرة عام 1976، ثم أعاد طبعه مرتين مع التنقيح والزيادة مرة عام 1988 ومرة عام 1997، وبيع منه في هذه الطبعات الثلاث أكثر من ربع مليون نسخة، وتحدث فيه عن الصور المختلفة السائدة من رؤية العالم في الغرب : المسيحية، الطبيعية، العدمية، وحدة الوجود الشرقية، فلسفة العصر الجديد، العدمية، ما بعد الحداثة. وفي الطبعة الرابعة التي صدرت عام 2004 تحدث في مقدمة الكتاب عن التحديثات التي أدخلها على الكتاب، وأشار إلى أمر كان يجب أن يضيفه إلى هذه الطبعة لكنه لم يفعل؛ لأنه لا يشعر أن بمقدوره القيام بهذه المهمة بالطريقة التي يجب أن تتم بها، وهو الحديث عن الرؤية الإسلامية للعالم، التي أصبح من الضروري عرضها بعد أحداث 11 أيلول 2001 في أمريكا، لأهما أصبحت تمسّ مساً وثيقاً حياة الناس حول العالم. يشير المؤلف بشيء من الاستغراب -والسرور طبعاً- إلى تعدد الجهات ونوعيات القراء الذين اهتموا بالكتاب حتى إنه ترجم إلى خمس عشرة لغة واعتبر من القراءات الأساسية في مساقات تعليمية متعددة في الفلسفة والأدب والتاريخ والدين وعلم اجتماع العلم، ولعل هذه الحقيقة تؤكد مصداقية إحدى الافتراضات التي بني عليها الكتاب، وهي أن القضايا الأساسية التي يهتم بها الناس -بوصفهم بشراً، أي بغض النظر عن جميع الاختلافات بينهم- ليس بينها حدود فاصلة ميزة لما، وهذه القضايا التي تتعلق بطبيعة الحقيقة الأساسية: الخالق أم الكون، وطبيعة الوجود الإنساني، وحقيقة الموت، ومعنى الحياة تتصل بالأدب مثل اتصالها بعلم النفس والدين والعلم. وعلى هذا الأساس عرض المؤلف المذكور كل رؤية من رؤى العالم السبعة التي حددها في كتابه من خلال إجابتها عن سبعة أسئلة تتعلق بالحقيقة الأساسية في الوجود، والكون، والإنسان، والموت، والمعرفة، وقيم الخير والشر، ومعنى التاريخ البشري. 
وبالرجوع إلى الموضوعات التي تبحث عن عنوان هذا المصطلح سنجد أها في بجملها ما يدخل تحت عنوان

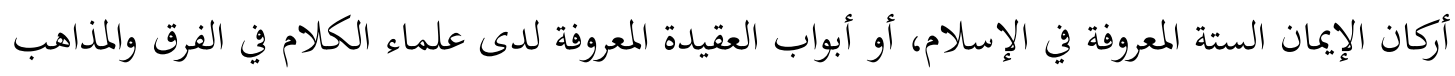
الإسلامية؛ أو الأصول الحمسة في الصياغة المعتزلية التي تميزت بإضافة أصل العدل؛ أو أصول الاعتقاد عند الشيعة الإثني عشرية التي تميزت بإضافة أصل الإمامة. إن وظيفة رؤية العالم في الأساس هو تزويدنا بالإطار العام الذي نفهم به كل شيء ونفهم أنفسنا أيضاً، وجعل فهمنا ضمن كل موحد، فكلما حاولنا أن نكون فهماً معيناً أو نصوغ نظرية لتفسير شيء ما، فإننا بالضرورة وبطبيعة عمل العقل نستخدم رؤيتنا للعالم. ولذلك فإن وظيفة رؤية العالم هي وظيفة معرفية. ودور المفكر المسلم المعاصر لا يقتصر على ضرورة استخدام رؤية العالم وجدة تحليل للأفكار والمواقف والأشخاص والمؤسسات، ليعرف أين وكيف تختلف رؤيتنا للعالم عن الرؤى الأخرى، بل عليه أن يجعل الرؤية الإسلامية للعالم معروفة لأصحاب الرؤى الأخرى، ليهلك من هلك عن بينة ويحيا من حيّ عن بينة! 